\title{
Spatially Resolved Spectroscopy to Confirm or Disprove Dual Active Galactic Nuclei
}

\author{
Rosalie C. McGurk ${ }^{1}$, Claire E. Max ${ }^{1}$, Anne Medling ${ }^{2}$ \\ and Gregory A. Shields ${ }^{3}$ \\ ${ }^{1}$ Astronomy Department and UCO-Lick Observatory, University of California, \\ Santa Cruz, CA 95064, USA, \\ Postbus 80000, NL-3508TA, Utrecht, the Netherlands \\ email: rmcgurk@ucsc.edu, max@ucolick.org \\ ${ }^{2}$ Research School of Astronomy and Astrophysics, Australian National University, \\ Mount Stromlo Observatory, Cotter Road, Weston Creek, ACT 2611, Australia \\ email: anne.medling@anu.edu. au \\ ${ }^{3}$ Astronomy Department, University of Texas, Austin, TX 78712, USA; \\ email: shieldsga@mail.utexas.edu
}

\begin{abstract}
When galaxies merge, gas accretes onto both central supermassive black holes. Thus, one expects to see dual active galactic nuclei (AGNs) in a fraction of galaxy mergers. Candidates for galaxies containing dual AGNs have been identified by the presence of double-peaked narrow [O III] emission lines and by high spatial resolution images of close galaxy pairs. $30 \%$ of double-peaked narrow [OIII] emission line SDSS AGNs have two spatial components within a $3^{\prime \prime}$ radius. However, spatially resolved spectroscopy is needed to confirm these galaxy pairs as systems with double AGNs. With the Keck 2 Laser Guide Star Adaptive Optics system and the OSIRIS near-infrared integral field spectrograph, we obtained spatially resolved spectra for SDSS J095207.62+255257.2, confirming that it contains a Type 1 and a Type 2 AGN separated by $4.8 \mathrm{kpc}\left(=1.0^{\prime \prime}\right)$. We performed similar integral field and long-slit spectroscopy observations of more spatially separated candidate dual AGNs and will report on the varied results. By assessing what fraction of radio-quiet double-peaked emission line SDSS AGNs are true dual AGNs, we can better constrain the statistics of dual AGNs and characterize physical conditions throughout these interacting AGNs.
\end{abstract}

Keywords. galaxies: active, galaxies: interactions, (galaxies:) quasars: emission lines, galaxies: nuclei

\section{Introduction}

One common explanation for active galactic nuclei (AGNs) invokes mergers of gas-rich galaxies; tidal forces can disturb the gas and funnel it into the centers of the merging galaxies, causing new bursts of star formation in the nuclear regions, increased fueling of the supermassive black hole(s), and their possible activation as an Active Galactic Nucleus (AGN) (Hernquist 1989; Kauffmann \& Haehnelt 2000; Hopkins et al. 2008).

If galaxy mergers trigger AGN activity, the nuclei of both galaxies should be active in at least some galaxy mergers. Hundreds of AGN pairs are known at $>15 \mathrm{kpc}$ separations (e.g. Hennawi et al. 2010; Green et al. 2010), while only $\sim 20$ spatially and spectrally confirmed pairs of AGNs are known with separations $<15 \mathrm{kpc}$ (commonly known as dual AGNs). At these separations, dual AGNs can be observed as double-peaked emission lines when the spectra are spatially unresolved (McGurk et al. 2012, Fu et al. 2012).

To confirm a potential dual AGN, spatially resolved spectroscopy is needed to prove that each resolved source has a unique AGN spectrum. Without spatially resolved 

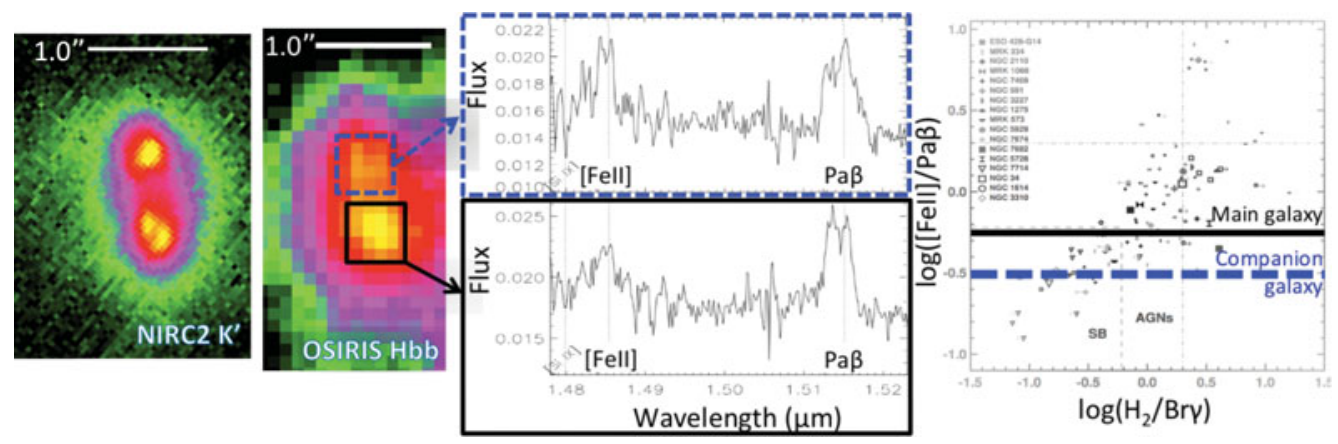

Figure 1. Keck NIRC2 and OSIRIS data for the galaxy and AGN J1108+0659 and its companion star-forming galaxy. Far Left: NIRC2 $K^{\prime}$ continuum image with the $1.0^{\prime \prime}$ scale bar showing $3.0 \mathrm{kpc}$ at the AGN's redshift. The main galaxy (bottom) with the AGN shows 2 small clumps, perhaps a bulge bisected with a dust lane; the companion galaxy (top) is located $0.73^{\prime \prime}$ (2.2 kpc) away. Middle Left: OSIRIS $H$-band continuum image showing that the 100 milliarcsec pixel scale used does not spatially resolve the two clumps in the main galaxy. Middle Right: Extracted spectra: top dashed blue box shows the star-forming companion galaxy, and bottom solid black box shows the AGN. While emission lines are detected in both galaxies, there is insufficient signal-to-noise to determine accurate line centers and velocity differences, especially of the double-peaks suggested in the main AGN spectrum. Far Right: Rodríguez-Ardila et al. near-infrared AGN/star-formation emission line diagnostic diagram, similar to the optical Baldwin-Phillips-Terlevich diagram. Rodríguez-Ardila et al. (2008) plot the emission line flux ratios [Fe II] $\lambda 1.2570 \mu \mathrm{m} / \mathrm{Pa} \beta$ vs. $\mathrm{H}_{2} \lambda 2.121 \mu \mathrm{m} / \mathrm{Br} \gamma$ star-forming galaxies and Type 1 and 2 Seyfert AGNs; dotted lines show the star-forming, AGN, and inconclusive regions of the diagram. Over this, we have plotted the $[\mathrm{Fe} \mathrm{II}] \lambda 1.2570 \mu \mathrm{m} / \mathrm{Pa} \beta$ line ratios of the main AGN and companion galaxy as, respectively, solid black and dashed blue lines (since we do not have observations of the $\mathrm{H}_{2} \lambda 2.121 \mu \mathrm{m}$ and $\mathrm{Br} \gamma$ lines). The original SDSS spectrum informs us that there is at least one AGN present in system, and the main galaxy appears to contain it. The companion galaxy appears to fall primarily in the star-forming region, so it is likely a star-forming galaxy.

spectroscopy to match the observed galaxies to their corresponding double-peaked emission lines, the double peaks of spectral lines may be due to a chance superposition of two objects, a recoiling SMBH (Bonning et al. 2007), jets interacting with the surrounding medium (Rosario et al. 2010; Veilleux et al. 2013), biconical or one-sided outflows from a single AGN (Fischer et al. 2011; Rupke \& Veilleux 2013), or rings of star formation. Similarly, without spatially resolved spectroscopy, the multiple bright cores imaged in or around one galaxy may be any of the previously listed scenarios, gravitationally lensed sources (Hennawi et al. 2006), or starbursts. High-energy X-ray observations are another unambiguous way to confirm true dual AGNs at wide $\left(>1^{\prime \prime}\right)$ separations, but they lack the spatial resolution to probe dual AGNs at smaller separations.

\section{Overview}

Recent studies have searched large extragalactic spectroscopic datasets such as the Sloan Digital Sky Survey (SDSS) to arrive at statistically useful samples of doublepeaked emission line AGNs. The catalogs of Smith et al. (2010), Wang et al. (2009), and Liu et al. (2010) create a well-defined sample of 340 candidate dual AGNs by selecting SDSS DR7 $\dagger$ spectroscopic AGNs that show a double-peaked [O III] $\lambda 5008$ line. Using the NIRC2 camerał and the Keck II Laser Guide Star Adaptive Optics (LGS AO) system

$\dagger$ http://www.sdss.org/dr7/

$\ddagger$ http://www2.keck.hawaii.edu/inst/nirc2/ 
(Wizinowich et al. 2006), Rosario et al. (2011), Fu et al. (2011, 2012), and McGurk et al. (in prep) undertook near-infrared (NIR) imaging of these candidate double AGNs. In total, 140 SDSS AGNs with double-peaked [O III] were imaged with NIRC2; 42 objects $(30 \%)$ have a companion within $3^{\prime \prime}$ of the primary galaxy.

Unfortunately, the imaging discussed above proves little about how the double emission line structure relates to presence of a companion. Spatially resolved spectroscopy is needed to map the spatial separation of the double-peaked emission lines and distinguish outflows from dual AGNs. We used the OH Suppressing InfraRed Integral field Spectrograph (OSIRIS) (Larkin et al. 2006) and the LGS AO system on the Keck II telescope to obtain spatially resolved spectroscopy for SDSS J091646.03+283526.7, SDSS J110851.04+065901.4, and SDSS J095207.62+255257.2 (respectively shortened to J0916+2835, J1108+0659, and $\mathrm{J} 0952+2552$ for the rest of the paper). The $0.100^{\prime \prime} /$ spatial pixel (spaxel) plate scale was used to maximize throughput.

We used a modified $\dagger$ version of the OSIRIS Data Reduction Pipeline (ODRP) $\mathrm{v} 2.3 \ddagger$ to process our images. Instead of using the standard mosaicking tools provided in the ODRP, we determined relative offsets between the images by fitting two-dimensional Lorentzian profiles to the main galaxy in each frame, and then input the relative offsets directly into the mosaicking module of the ODRP 9 . Finally, we extracted and combined spectra from the five spatial pixels with the highest signal-to-noise ratio $(\mathrm{S} / \mathrm{N})$ of both the main galaxy, the companion galaxy, and any interesting local features, as shown in Figures 1 and 2 for J1108+0659 and

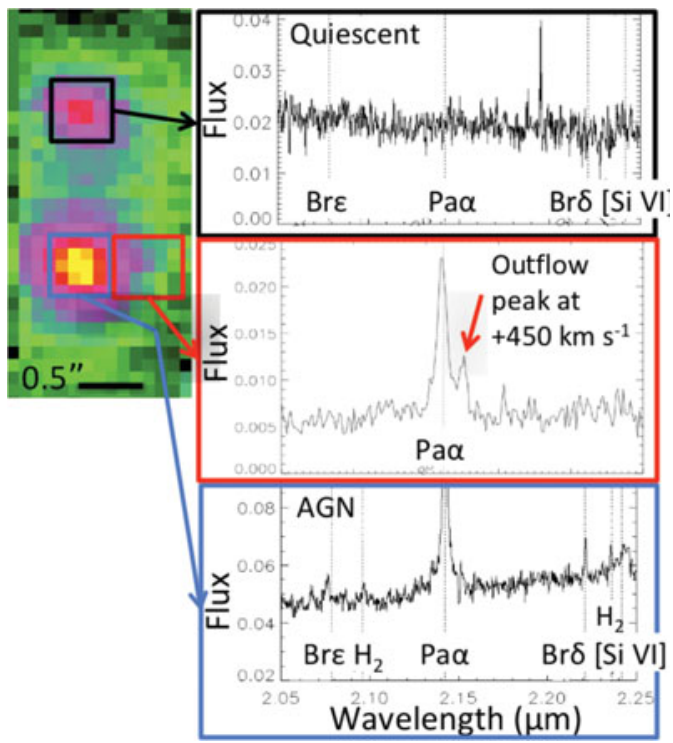

Figure 2. Keck OSIRIS data for the galaxy and AGN J0916+2835, its outflow, and its companion. Left: OSIRIS $K$-band continuum image of the galaxy (bottom) and its fainter companion (top). Right: Extracted spectra: top black box shows the quiescent companion galaxy, bottom blue box shows the AGN, middle red box shows the outflow. Multiple lines are observed in the AGN at the SDSS redshift of 0.142 . In a continuum-less region $1.2 \mathrm{kpc}$ West of the AGN (middle red box), we observe a second $\mathrm{Pa} \alpha$ peak at $+450 \mathrm{~km} \mathrm{~s}^{-1}$, consistent with the SDSS [O III] $\lambda 5008$ velocity difference of $425 \mathrm{~km} \mathrm{~s}^{-1}$. This ionized gas emission without stellar continuum is well explained by an outflow. The companion galaxy (top, black box) contains no significant emission lines (ignoring one cosmic ray). It is either a quiescent galaxy or a foreground or background object.

J0916+2835, respectively. Additionally, an OSIRIS image can be created by summing up the flux over all of the wavelengths (Figure 1, middle-left panel, and Figure 2, left panel).

\section{Discussion}

For the in-depth discussion of J1108+0659 and J0916+2835, please see the captions of Figures 1 and 2, respectively. For an in-depth discussion of J0952+2552, please see

$\dagger$ Correcting for $\mathrm{a} \sim 6 \AA$ shift of the previous wavelength calibration Wright 2011.

$\ddagger$ http://irlab.astro.ucla.edu/osiris/pipeline.html

ๆ See http://irlab.astro.ucla.edu/osiriswiki/dokuphp?id=mosaic_with_a_list_of_offsets for a detailed explanation. 
McGurk et al. (2011). In J1108+0659, both the main galaxy and the companion galaxy show [Fe II] $\lambda 1.2570 \mu \mathrm{m}$ and $\mathrm{Pa} \beta$ emission lines, although the lines lack signal-to-noise to conclude their precise wavelength centers and velocity differences. Independent of the spectral resolution, our high spatial resolution allows us to independently determine that the main galaxy contains the AGN detected by the SDSS at the redshift of 0.182 while the emission lines from the companion galaxy are at the same redshift and appear to be due to star-formation. In J0916+2835, multiple emission lines confirm that there is an AGN at the SDSS redshift of 0.142 . However, the companion galaxy contains no significant emission lines, and is either a quiescent galaxy or a foreground or background object. Excitingly, in a continuumless region $1.2 \mathrm{kpc}$ West of the AGN, we observe a second $\mathrm{Pa} \alpha$ at $+450 \mathrm{~km} \mathrm{~s}^{-1}$ with respect to the central AGN, consistent with the SDSS [O III] $\lambda 5008$ velocity difference of $425 \mathrm{~km} \mathrm{~s}^{-1}$. Ionized gas emission without stellar continuum is well-explained by the presence of an outflow; a second, less likely possibility is the presence of a supermassive black hole retaining its accretion disk and narrow line region, without any nearby stars to supply continuum light.

These results and more will be discussed in McGurk et al. (in prep.).

\section{References}

Bonning, E. W., Shields, G. A., \& Salviander, S. 2007, ApJL, 666, L13

Fu, H., Myers, A. D., Djorgovski, S. G., \& Yan, L. 2011, ApJ, 733, 103

Fu, H., Yan, L., Myers, A. D., Stockton, A., Djorgovski, S. G., Aldering, G., \& Rich, J. A. 2012, ApJ, 745, 67

Green, P. J., Myers, A. D., Barkhouse, W. A., Mulchaey, J. S., Bennert, V. N., Cox, T. J., \& Aldcroft, T. L. 2010, ApJ, 710, 1578

Hennawi, J. F., Myers, A. D., Shen, Y., Strauss, M. A., Djorgovski, S. G., Fan, X., Glikman, E., Mahabal, A., Martin, C. L., Richards, G. T., Schneider, D. P., \& Shankar, F. 2010, ApJ, 719,1672

Hernquist, L. 1989, Nature, 340, 687

Hopkins, P. F., Hernquist, L., Cox, T. J., \& Kereš, D. 2008, ApJS, 175, 356

Kauffmann, G. \& Haehnelt, M. 2000, MNRAS, 311, 576

Larkin, J., Barczys, M., Krabbe, A., Adkins, S., Aliado, T., Amico, P., Brims, G., Campbell, R., Canfield, J., Gasaway, T., Honey, A., Iserlohe, C., Johnson, C., Kress, E., LaFreniere, D., Lyke, J., Magnone, K., Magnone, N., McElwain, M., Moon, J., Quirrenbach, A., Skulason, G., Song, I., Spencer, M., Weiss, J., \& Wright, S. 2006, in Society of Photo-Optical Instrumentation Engineers (SPIE) Conference Series, Vol. 6269, 62691A

Liu, X., Shen, Y., Strauss, M. A., \& Greene, J. E. 2010, ApJ, 708, 427

McGurk, R. C., Max, C. E., Rosario, D. J., Shields, G. A., Smith, K. L., \& Wright, S. A. 2011, ApJL, 738, 2

Rodríguez-Ardila, A., Riffel, R., \& Carvalho, E. A. 2008, in Revista Mexicana de Astronomia y Astrofisica Conference Series, Vol. 32, 77-79

Rosario, D. J., McGurk, R. C., Max, C. E., Shields, G. A., \& Smith, K. L. 2011, ApJ, 739, 44

Rosario, D. J., Shields, G. A., Taylor, G. B., Salviander, S., \& Smith, K. L. 2010, ApJ, 716, 131

Rupke, D. S. N. \& Veilleux, S. 2013, ApJL, 775, 15

Smith, K. L., Shields, G. A., Bonning, E. W., McMullen, C. C., Rosario, D. J., \& Salviander, S. $2010, A p J, 716,866$

Wang, J.-M., Chen, Y.-M., Hu, C., Mao, W.-M., Zhang, S., \& Bian, W.-H. 2009, ApJL, 705, L76

Wizinowich, P. L., Le Mignant, D., Bouchez, A. H., Campbell, R. D., Chin, J. C. Y., Contos, A. R., van Dam, M. A., Hartman, S. K., Johansson, E. M., Lafon, R. E., Lewis, H., Stomski, P. J., Summers, D. M., Brown, C. G., Danforth, P. M., Max, C. E., \& Pennington, D. M. 2006, PASP, 118, 297

Wright, S. A. 2011, private communication 